\title{
Interview
}

\section{Pflege muss endlich Chefsache werden}

\author{
Überall fehlen Pflegekräfte. Dennoch wird das Pflegestellen-Förderpro- \\ gramm der Bundesregierung bisher nur verhalten genutzt. Woran liegt \\ das? Wir fragten nach beim Vorsitzenden des Bundesverbands Pflegema- \\ nagement Peter Bechtel.
}

? Es gibt Geld für neue Pflegestellen und die Krankenhäuser rufen es kaum ab. Wie erklären Sie sich diese Zurückhaltung?

Bechtel: Das hängt sicher damit zusammen, dass die Krankenhäuser ihre Zahlen zum Personalbestand so aufbereiten mussten, dass sie der sogenannten Stichtagsprüfung standhalten. Viele haben das für das Jahr 2016 nicht geschafft. Ein ähnliches Förderprogramm im Jahr 2009 ist anfangs ebenfalls schleppend angelaufen. Ein weiterer Grund könnte darin liegen, dass manche Kliniken entsprechend der Vorgaben nur verschwindend wenige neue Planstellen bekommen würden. Ein Antrag wird dann möglicherweise gar nicht erst gestellt.

? Wer die Fördergelder in Anspruch nimmt, muss die korrekte Verwendung nachweisen. Fürchten sich die Kliniken vor Kontrolle?

Bechtel: Das spielt sicherlich eine Rolle. Die Zweckgebundenheit der zur Verfügung gestellten Mittel ist im Förderprogramm eindeutig geregelt. In vielen anderen Fällen können und werden die
Mittel dort eingesetzt, wo die Geschäftsführung sie einsetzen will. Die Transparenz ist daher ein sehr wichtiger Faktor. Nur so ist garantiert, dass das Geld wirklich in zusätzliche Stellen für die Pflege fließt und nicht fehlgeleitet wird für längst überfällige Sanierungen oder die Beschaffung von Großgeräten.

? Geht die Strategie der Bundesregierung auf, mit dem Programm die Pflege am Bett zu stärken?

Bechtel: Grundsätzlich halte ich es für den richtigen Weg, die Pflege am Bett zu stärken und damit eine qualitativ angemessene Patientenversorgung anzustreben. Leider stehen in vielen Teilen unseres Landes auf dem Arbeitsmarkt nicht die entsprechenden zusätzlichen Pflegekräfte zur Verfügung, um diese einzustellen. Dies sollte aber nicht dazu führen, das Förderprogramm auszusetzen. Vielmehr müssen wir eine konzertierte Aktion starten, um den Pflegeberuf attraktiv zu gestalten. Leider ist hier mit dem mehr als halbherzigen Ansatz zur generalistischen Ausbildung eine historische Chance vertan worden!

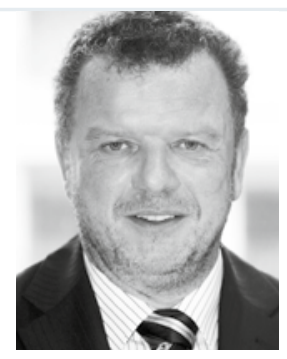

Peter Bechtel

Pflegedirektor am Universitäts-Herzzentrum FreiburgBad Krozingen
? Was müsste noch geschehen, um den Personalmangel $z$ u bekämpfen?

Bechtel: Alle Akteure müssen an einem Strang ziehen, um dem Personalmangel zumindest mittelfristig begegnen zu können. Grundlage dafür könnte ein „Bundespflegeplan“ sein, der all die Faktoren beschreibt, die einer dringenden $\mathrm{Be}$ arbeitung bedürfen, um die Attraktivität des Berufs zu steigern. Neben einer besseren Personalausstattung gehört dazu auch die Frage nach einer adäquaten Vergütung, insbesondere auch bei abgeschlossenem Studium. Die Frage nach vorbehaltenen Tätigkeiten für die Profession Pflege spielt hier ebenso eine Rolle wie die Begegnung auf Augenhöhe mit den anderen Professionen. Aus meiner Sicht ist es längst überfällig, dass unsere Kanzlerin das Thema Pflege - und damit meine ich in erster Linie die Profession zur "Chefsache" macht!

Das Interview führte Nicoletta Eckardt.

Das komplette Interview finden Sie hier oder unter www.springerpflege.de

\section{Gründungskonferenz}

\section{Bundespflegekammer: Es geht los}

— Die Vorbereitungen für eine Bundespflegekammer haben begonnen. Der Deutsche Pflegerat (DPR) und die Landespflegekammer Rheinland-Pfalz haben beschlossen, eine Gründungskonferenz einzurichten. Diese soll innerhalb von höchstens einem Jahr alle Vorbereitungen für eine Bundespflegekammer als Dach bestehender und zukünftiger Landespflegekammern treffen. Bisher hat bereits die Landespflege- kammer Rheinland-Pfalz ihre Arbeit aufgenommen, Initiativen gibt es derzeit in Schleswig-Holstein, Niedersachsen, Nordrhein-Westfalen und Baden-Württemberg. Die Bundespflegekammer soll eine "gemeinsame und einheitliche Selbstverwaltung aller professionellen Pflegeberufe auf Bundesebene" sicherstellen, heißt es.

www.deutscher-pflegerat.de

\section{Gestellung von Rotkreuzschwestern DRK-Gesetz geändert}

— Gute Nachrichten für den Verband der Schwesternschaften: Die Änderung des DRK-Gesetzes ist im BGBI. veröffentlicht. Damit wird die unbefristete Gestellung von Rotkreuzschwestern weiterhin möglich sein. Die im Arbeitnehmerüberlassungsgesetz vorgesehene Überlassungshöchstdauer von 18 Monaten wird nicht mehr gelten.

www.rotkreuzschwestern.de 